\title{
Modalités de prise en charge du patient asthmatique en chirurgie buccale
}

\section{Modalities of management of asthmatic patients in dental surgery}

\author{
HERVÉ MOIZAN*, ANDRÉ LAGARDE**, ALAIN HALOUN***
}

\begin{abstract}
RÉSUMÉ
Dans notre spécialité, les conditions d'apparition d'une crise d'asthme sont multiples. Le stress, la douleur, l'utilisation d'anesthésiques locaux, l'infection, la prescription médicamenteuse et l'exposition à certains pneumallergènes comme le latex et les poussières de fraisage peuvent favoriser celle-ci. L'asthme est responsable d'environ 2000 décès par an en France et justifie 40000 hospitalisations par an. Le tiers des décès survient dans la première demi-heure après le début des signes et $60 \%$ en dehors de l'hôpital.

La pathologie asthmatique semble en constante augmentation ; nombreux sont les patients sous-médicalisés et sous-traités ; l'observance des traitements est souvent partielle. L'application du principe de prudence se justifie afin de conduire de façon rationnelle et en toute sécurité un acte de chirurgie buccale chez ces patients. L'objet de notre article est de systématiser la conduite à tenir et de proposer une stratégie pré, per- et post-opératoire. (Med Buccale Chir Buccale 2003; 9: 37-48)
\end{abstract}

mots clés : Asthme, Chirurgie buccale

médecine buccale chirurgie buccale

VOL. $9, \mathrm{~N}^{\circ} 1$ 2003

page 37

\section{SUMMARY}

Various factors may predispose to asthma attacks in the context of dental surgery. Stress, pain, the use of local anaesthetics, infection, prescription of drugs and exposure to certain respiratory allergens such as latex and reaming dust can trigger an asthma attack. Asthma is responsible for about 2,000 deaths and 40,000 hospitalizations each year in France. One third of deaths occur during the first half hour after onset of the symptoms and $60 \%$ of deaths occur outside hospital.

The prevalence of asthma appears to be constantly increasing; many patients are insufficiently medicalized and undertreated and compliance with treatment is often only partial. A cautious approach is therefore justified to ensure rational and perfectly safe oral surgery in these patients. The objective of this article is to define the management of these patients and propose an adapted preoperative, intraoperative and postoperative strategy. (Med

\section{Buccale Chir Buccale 2003; 9: 37-48)}

key words : Asthma, Oral surgery

\footnotetext{
* Service d'Odontologie, CHR Metz

** Service d'Odontologie chirurgicale et restauratrice, CHU Hôtel Dieu Nantes

*** Service de Pneumologie, CHU Laennec Nantes
}

Demande de tirés à part :

Hervé MOIZAN : Service d'Odontologie CHR Metz-Thionville Hôpital N-D de Bon Secours 1, place Philippe de Vigneulles BP 81065 57038 Metz cedex 01 
médecine buccale chirurgie buccale

VOL. $9, \mathrm{~N}^{\circ} 1$ 2003

page 38

\section{INTRODUCTION}

L' asthme est plus que jamais une maladie d'aujourd'hui et de demain. Cette pathologie ne mérite pas sa réputation d'affection banale, voire bénigne. La fréquence de celle-ci et sa gravité n'ont cessé d'augmenter au cours des dix dernières années. La prévalence de l'asthme est élevée : 5 à $7 \%$ des adultes, et 10 à $15 \%$ des adultes jeunes entre 20 et 24 ans. En France la mortalité est globalement stable depuis 1990, autour de 2000 décès annuels, mais elle augmente discrètement dans la tranche des sujets jeunes de 5 à 34 ans [1]. Des études épidémiologiques montrent que le diagnostic d'asthme est connu dans seulement 30 à $50 \%$ des cas. Comme pour toute affection chronique, il existe chez de nombreux patients, un déni de l'affection conduisant à une non observance des thérapeutiques [2]. Les patients ne font pas toujours l'objet d' évaluation et de réévaluation sur le plan médical. Les traite- ments, pour une partie des malades, ne sont pas conformes aux recommandations ainsi $28 \%$ des asthmatiques présentant un asthme persistant modéré et jusqu'à $17 \%$ des malades atteints d'un asthme persistant sévère ne bénéficient pas d'une corticothérapie inhalée, base du traitement de fond dans toutes les recommandations internationales [2,3,4]. Toutes ces observations doivent attirer la vigilance de l'odontologiste qui prend en charge un patient asthmatique. Le malade doit être considéré comme potentiellement à risque si un certain nombre de conditions ne sont pas réunies pour conduire l'acte chirurgical avec des exigences de sécurité optimale. Cet article a pour objectif de rappeler les aspects physiopathologiques, les thérapeutiques actuelles ainsi que les précautions à prendre chez ces patients afin d'éviter toute interférence lors d'un acte chirurgical en odonto-stomatologie ou d'une prescription médicamenteuse.

Tableau 1 : Paliers thérapeutiques de l'asthme d'après le National Heart, Lung, and Blood Institut [8]. Classification of asthma by severity and treatments from National Heart, Lung and Blood Institut [8].

\begin{tabular}{|c|c|c|c|c|}
\hline STADE & 1 & 2 & 3 & 4 \\
\hline ASTHME & Intermittent & Persistant léger & Persistant modéré & Persistant sévère \\
\hline $\begin{array}{c}\text { SYMPTÔMES } \\
\text { AVANT } \\
\text { TRAITEMENT }\end{array}$ & $\begin{array}{l}\text { Symptômes }<2 \text { fois/ } \\
\text { semaine } \\
\text { Symptômes } \\
\text { nocturnes }<2 \text { fois/ } \\
\text { mois } \\
\text { DEP normal entre } \\
\text { les crises } \\
\text { VEMS - DEP : } \geq \\
80 \% \text { de la valeur } \\
\text { théorique } \\
\text { Variabilité } \\
\text { VEMS-DEP }<20 \%\end{array}$ & $\begin{array}{l}\text { Symptômes }>2 \text { fois/ } \\
\text { semaine et }<1 \text { fois/ } \\
\text { jour } \\
\text { Symptômes } \\
\text { nocturnes }>2 \text { fois/ } \\
\text { mois } \\
\text { VEMS-DEP : } \geq 80 \% \\
\text { de la valeur } \\
\text { théorique } \\
\text { Variabilité VEMS- } \\
\text { DEP : } 20 \text { à } 30 \%\end{array}$ & $\begin{array}{l}\text { Symptômes } \\
\text { quotidiens } \\
\text { Crises retentissant / } \\
\text { activité et sommeil } \\
\text { Asthme nocturne > } \\
1 \text { fois / semaine } \\
\text { Usage fréquent de } \\
\text { B2 immédiats } \\
\text { VEMS-DEP : } 60 \text { à } \\
80 \% \text { de la valeur } \\
\text { théorique } \\
\text { Variabilité VEMS- } \\
\text { DEP > } 30 \%\end{array}$ & $\begin{array}{l}\text { Symptômes } \\
\text { permanents } \\
\text { Exacerbations } \\
\text { fréquentes } \\
\text { Asthme nocturne } \\
\text { fréquent } \\
\text { Activité physique } \\
\text { limitée / symptômes } \\
\text { VEMS-DEP } \leq 60 \text { \% } \\
\text { de la valeur } \\
\text { théorique } \\
\text { Variabilité } \\
\text { VEMS-DEP> } 30 \%\end{array}$ \\
\hline TRAITEMENT & $\begin{array}{l}\text { Pas de traitement } \\
\text { continu. } \\
\text { B2-agonistes à } \\
\text { action immédiate, à } \\
\text { la demande mais < } \\
1 \text { fois / semaine }\end{array}$ & $\begin{array}{l}\text { Corticoïdes inhalés - } \\
\text { cromones ou ß2- } \\
\text { agonistes quotidiens } \\
\text { sans dépasser } 3 \text { à } \\
4 \text { fois/jour }\end{array}$ & $\begin{array}{l}\text { Corticoïdes inhalés + } \\
\text { bronchodilatateur à } \\
\text { action prolongée + } \\
\text { B2-agonistes à } \\
\text { action immédiate, } \\
\text { à la demande }\end{array}$ & $\begin{array}{l}\text { Corticoïdes inhalés + } \\
\text { bronchodilatateur à } \\
\text { action prolongée + } \\
\text { corticoïde oral + } \\
\text { ß2-agonistes à } \\
\text { action immédiate, } \\
\text { à la demande }\end{array}$ \\
\hline
\end{tabular}




\section{DEFINITION DE L'ASTHME}

L'asthme est une pathologie inflammatoire chronique des bronches qui se traduit par la survenue de crises dyspnéiques paroxystiques, réversibles spontanément ou sous l'effet des traitements, et causée par une obstruction bronchique secondaire à des mécanismes immunologiques ou autres [5]. Ces crises peuvent être sévères, voire mortelles. Dans les périodes d'inter-crises, le malade n'a aucun trouble, au moins durant les premières années de la maladie. Néanmoins, des anomalies anatomiques, biochimiques et physiopathologiques subsistent [6]. L'asthme n'est pas une maladie au sens pasteurien du terme mais un syndrome multifactoriel d'origine génétique, dont l'expression est largement fonction de facteurs acquis, souvent liés à l'environnement.

Le diagnostic repose sur la mise en évidence d'un trouble ventilatoire obstructif, à un moment ou à un autre, puisque la pathologie est caractérisée par des symptômes intermittents et imprévisibles. Plusieurs méthodes sont utilisées pour mettre en évidence le trouble ventilatoire obstructif, telle que la spirométrie avec notamment le volume expiratoire maximal par seconde (VEMS) et la mesure du débit expiratoire de pointe (DEP). Les différentes recommandations internationales apprécient la sévérité de l'asthme en tenant compte des symptômes, de la consommation médicamenteuse (B2-agonistes) et de la fonction pulmonaire de base et de sa variabilité dans le temps (VEMS, DEP). Le tableau 1 reprend la classification des différents stades de la maladie et des protocoles thérapeutiques associés chez l'adulte et les enfants au-delà de 5 ans [7].

\section{PHYSIOPATHOLOGIE}

Au cours de la dernière décennie, des progrès décisifs ont été accomplis dans la compréhension des mécanismes physiopathologiques de l'asthme bien qui ceux-ci soient complexes car multifactoriels. Trois mécanismes permettent de mieux comprendre la pathologie $[5,6,9,10]$.

\section{- L'inflammation bronchique}

Les mécanismes de l'inflammation bronchique sont encore mal compris et les théories mul- tiples: inflammation allergique, inflammation neurogène, anomalie de la présentation antigénique, inflammation mastocytaire et/ou éosinophilique et/ou lymphocytaire. Le dérèglement initial est inconnu. II n'existe pas une cellule ou un médiateur impliqué dans la réaction inflammatoire mais un ensemble de cellules et de médiateurs. Les lésions histologiques constatées sont d'autant plus nettes que l'on considère les couches les plus proches de la lumière bronchique. L'épithélium est la tunique la plus lésée. Les médiateurs libérés (histamine, leucotriènes, cytokines...) dénudent l'épithélium, augmentent le spasme bronchique et entraînent œdème et inflammation de la sous-muqueuse diminuant alors le calibre de la lumière bronchique. Cette inflammation est en corrélation directe avec la sévérité de l'asthme et le degré d'hyperréactivité bronchique. Le système nerveux autonome intervient dans la régulation du tonus du muscle lisse bronchique, mais également dans la dégranulation mastocytaire. L'innervation des voies aériennes est relativement complexe ; le système paraympathique est bronchoconstricteur, son médiateur représenté par l'acétylcholine agit sur les récepteurs muscariniques M3 du muscle lisse bronchique ; le système sympathique est bronchodilatateur, ses médiateurs - catécholamines, adrénaline et noradrénaline - actionnent les récepteurs $B 2$ du muscle lisse. A coté des deux systèmes décrits, un système complexe dit non adrénergique non cholinergique (NANC), mis en évidence plus récemment chez l'homme, a une double action bronchodilatatrice et bronchoconstrictrice. Ce dernier faisant intervenir des peptides divers comme le Vasoactive Intestinal Peptide (VIP), la substance $\mathrm{P}(\mathrm{SP})$, les tachykinines, le Calcitonin Gen Related Peptide (CGRP) et le Neuropeptide Y (NPY).

\section{- L'obstruction bronchique ou bronchocons- triction}

Le muscle lisse bronchique entoure l'ensemble des voies aériennes. Sa contraction est responsable d'un trouble ventilatoire obstructif diffus, d'intensité variable selon le degré de réponse du muscle au stimulus. Cette contrac- médecine

buccale

chirurgie

buccale

VOL. $9, \mathrm{~N}^{\circ} 1$ 2003

page 39 
médecine buccale chirurgie buccale

VOL. $9, \mathrm{~N}^{\circ} 1$ 2003

page 40 tion est réversible, soit spontanément, soit sous l'effet de drogues telles les B2-agonistes, les vagolytiques, la théophylline et les antileucotriènes. Une hypertrophie du muscle lisse est par ailleurs responsable d'une augmentation de l'épaisseur de la paroi, donc d'une réduction de calibre de la lumière bronchique, d'autre part d'une augmentation de la réactivité bronchique. Ce muscle lisse bronchique pourrait par ailleurs avoir un rôle actif dans la synthèse et la libération de médiateurs chimiques, pro-inflammatoires et bronchoconstricteurs.

\section{- Les bouchons muqueux}

Les glandes à mucus sont également hypertrophiées et participent à l'augmentation de l'épaisseur de la paroi bronchique. Ces glandes sont retrouvées dans les bronchioles périphériques chez le sujet asthmatique alors qu'elles en sont habituellement absentes.

\section{FORMES CLINIQUES}

L'asthme paroxystique est caractérisé cliniquement par la survenue d'épisodes de dyspnée sifflante, d'oppression thoracique et de toux. La crise d'asthme est l'élément à la base du syndrome asthme. Elle s'installe rapidement, volontiers la nuit ou au réveil. Elle se caractérise par l'apparition de prodromes : sensation de malaise général, picotement laryngé, crise d'éternuements, toux sèche. La phase d'état se subdivise en deux phases:

La phase sèche se caractérise par une dyspnée à type de bradypnée expiratoire, des sifflements importants (râles sibilants), une anxiété, un pouls accéléré, un thorax globuleux.

La phase sécrétoire qui survient 30 à 60 minutes après la phase sèche, dure parfois plusieurs heures. La dyspnée s'apaise, une toux continue apparait avec expectoration de mucus dense (crachats "perlés » de Laennec). Cette crise peut céder en quelques minutes après inhalation de B2-agonistes.

L'asthme à dyspnée continue se traduit comme son nom l'indique par la survenue de crises paroxystiques, parfois nocturnes, soulagées par les bronchodilatateurs, et sur un fond de dyspnée continue notamment à l'effort. II est retrouvé des râles sibilants en période intercritique. II succède à un asthme paroxystique ancien et réalise un tableau de bronchopneumopathie chronique obstructive (BPCO).

\section{Exacerbation de l'asthme et asthme instable}

L'exacerbation de l'asthme correspond à la persistance des symptômes pendant une durée plus longue (1 à plusieurs jours). Elle peut survenir à tous les stades de sévérité, y compris lorsque l'asthme est contrôlé. Elle s'accompagne d'une aggravation de l'obstruction bronchique qui reste incomplètement levée par les B2 agonistes. L'attaque d'asthme est une exacerbation. Elle se définit comme la succession de crises, pendant plusieurs jours ; entre les crises, la dyspnée peut être présente sur un mode mineur ou absente.

L'asthme instable est une condition favorisant la survenue de l'asthme aigu grave. L'asthme aigu grave (appelé aussi état de mal asthmatique) peut mettre immédiatement le pronostic vital en jeu. Les crises se répètent, en s'intensifiant progressivement ; elles résistent aux différents traitements. Elle peut compliquer ou succéder en quelques heures à la crise d'asthme classique et constitue une urgence médicale. Les critères de l'asthme instable sont importants à connaître car ils imposent de manière urgente un renforcement du traitement de fond (Tableau 2).

Tableau 2 : Critères de l'asthme instable. Criteria of unstable asthma.

- Augmentation de la fréquence des crises

- Moindre sensibilité des crises aux bronchodilatateurs

- Grandes variations diurnes de l'obstruction bronchique

- Aggravation au petit matin (morning dip)

- Aggravation progressive de l'obstruction bronchique jugée par la baisse du DEP

- Augmentation progressive de la consommation de B2-agonistes 


\section{TRAITEMENT}

Le traitement de fond doit suivre les recommandations internationales et être adapté à chaque patient de façon spécifique selon le contexte clinique, psychologique et l'environnement. La cible prioritaire est le traitement de l'inflammation des voies aériennes, d'ou la priorité aux anti-inflammatoires inhalés [10-12].

\section{Les anti-inflammatoires}

- Les corticoïdes, médication primordiale pour le traitement de l'asthme au long cours, agissent en diminuant la perméabilité de l'endothélium aux cellules inflammatoires en réduisant l'expression des molécules d'adhésion, en inhibant la synthèse des médiateurs chimiques bronchoconstricteurs, en améliorant la clairance mucociliaire et en interrompant la mucosécrétion. On distingue les corticoïdes administrés par voie générale et les corticoïdes inhalés.

La corticothérapie orale (Cortancyl ${ }^{\circledR}$, Solupred $₫$ ) constitue le plus puissant traitement de l'asthme par son action drastique sur l'inflammation. En association au traitement inhalé à dose maximale, elle est envisagée seulement en dernier recours, en raison des effets secondaires importants lors de toute utilisation prolongée.

Les corticoïdes inhalés restent les seules substances capables de réduire de manière significative et durable l'hyperréactivité bronchique. Parmi les molécules employées, citons la béclométhasone (Bécotide $\AA$ ), la budésonide (Pulmicort $@$ ) et la fluticasone

(Flixotide $\AA$ ). Leur efficacité est spectaculaire, ils constituent la base du traitement de fond. Ils possèdent une action dose-dépendante. Ils peuvent être utilisés en cas d'asthme léger, à faible dose (inférieure à $1000 \mu \mathrm{g} . \mathrm{j}^{-1}$ ). Ils sont indispensables en cas d'asthme modéré ou

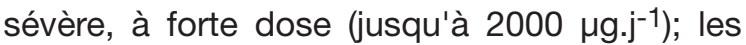
effets secondaires sont essentiellement locaux aux doses préconisées : candidose buccale et raucité de la voix. Ces effets indésirables sont prévenus par le rinçage de la cavité buccale ou l'ingestion d'un verre d'eau après la prise. A très fortes doses, des effets cutanés et osseux peu- vent être observés exceptionnellement, mais la réponse surrénalienne est toujours conservée. II faut noter que les corticoïdes ont une latence d'action d'au moins deux heures quel que soit le mode d'administration (y compris IV) [3].

( inflammatoires non stéroïdiens. Ils s'adressent aux asthmes légers ou modérés, après contrôle de la fonction pulmonaire. Leur action est préventive et différée de deux à quatre semaines. Ils ne possèdent pas d'effets secondaires. Le plus connu et le plus prescrit est le nédocromil sodique (Tilade $\AA$ ) qui est 4 à 10 fois plus actif que le Lomudal $\AA$, première molécule à avoir été introduite en 1970. L'effet anti-inflammatoire est beaucoup plus modeste que celui des corticoïdes. Ces médicaments agiraient sur les mastocytes et les basophiles comme «stabilisateurs" de membrane.

- Les antileucotriènes sont autorisés dans le traitement de fond de l'asthme persistant léger. Disponibles actuellement sous la forme orale (montelukast, Singulair $\AA$ ), ils sont dérivés des phospholipides membranaires soumis à l'action de phospholipases. L'acide arachidonique libéré est métabolisé par la voie de la cyclo-oxygénase vers les prostaglandines ou par la voie de la 5-lipo-oxygénase vers les leucotriènes ; ces derniers sont des médiateurs de la bronchoconstriction. D'efficacité inégale selon les patients, ils ont également un intérêt dans l'asthme cortico-résistant.

\section{Les bronchodilatateurs}

Ils sont définis par leur aptitude à corriger rapidement l'obstruction bronchique. Le muscle lisse est le site d'action préférentiel de cette classe médicamenteuse. Ils constituent un traitement d'appoint et peuvent être diversement associés aux corticoïdes inhalés.

- Les sympathomimétiques adrénergiques B2agonistes sont les bronchodilatateurs les plus puissants. Le récepteur $B$-adrénergique est une glycoprotéine membranaire ; il existe au moins trois sous-types $(B 1, B 2, B 3)$ dont la répartition \begin{tabular}{l}
$\begin{array}{l}\text { médecine } \\
\text { buccale } \\
\text { chirurgie } \\
\text { buccale }\end{array}$ \\
\hline VOL. $9, N^{\circ} 1$ \\
2003 \\
\hline page 41
\end{tabular} 
médecine

buccale

chirurgie

buccale

VOL. $9, \mathrm{~N}^{\circ} 1$ 2003

page 42 est la suivante : les récepteurs $\beta 1$ sont présents au niveau cardiaque, avec également quelques récepteurs $B 2$; les récepteurs $B 2$ sont répartis dans le muscle lisse bronchique exclusivement; les récepteurs $B 3$ sont retrouvés dans le muscle lisse du cœur et du tube digestif, et sur les cellules graisseuses.

La stimulation des récepteurs $B 2$ active une protéine kinase $A$ via le métabolisme de l'AMPcyclique. Les B2-mimétiques relâchent le muscle lisse bronchique; ils inhibent la libération d'acétylcholine par les terminaisons nerveuses, pourraient diminuer l'œdème bronchique en agissant sur les veinules post-capillaires et augmentent les sécrétions bronchiques. Enfin, ils améliorent la clairance mucociliaire et diminuent la libération des médiateurs mastocytaires. Ils n'ont pas d'effet anti-inflammatoire.

Les B2-agonistes de longue durée d'action sont devenus les bronchodilatateurs de référence en association aux corticoïdes inhalés. Les B2agonistes de courte durée d'action n'ont plus actuellement leur place dans le traitement de fond et ils sont presque exclusivement réservés au traitement de la crise.

Ces médicaments peuvent être administrés par voie veineuse, sous-cutanée, per os ou par voie inhalée. La voie veineuse (Salbutamol $\AA$ ) est utilisée en réanimation bien qu'on ait tendance à la remplacer par des nébulisations. L'administration sous-cutanée (Bricanyl $®$ ) peut être faite par le malade lui-même ou un de ses proches en attendant l'arrivée du médecin.

Les B2-mimétiques administrés par aérosols doseurs constituent la pierre angulaire du traitement symptomatique ; ils sont inhalés à la demande ou de manière régulière pour offrir une efficacité bronchodilatatrice aussi stable que possible. La technique d'inhalation doit être enseignée aux malades, en ayant éventuellement recours à une chambre d'inhalation qui permet une meilleure pénétration du principe actif sur l'ensemble des voies aériennes supérieures. L'autohaler est déclenché par le malade lui-même ce qui permet une meilleure coordination main-bouche.

Des B2-agonistes à longue action sont disponibles en aérosols doseurs (Serevent ${ }^{\circledR}$,
Foradil $\left.{ }^{\circledR}\right)$; ils ont une durée d'action de 12 heures. II existe également des B2-agonistes oraux de longue durée d'action $\left(\mathrm{Oxeol} \circledast{ }^{\circledR}\right)$ utilisés pour faciliter la compliance au traitement. Ces préparations ne constituent pas le traitement de la crise mais seront employées au long cours. Les effets secondaires de ces médications sont la tachycardie et des tremblements.

- La théophylline est le plus ancien médicament de l'asthme ; il a été introduit en 1937 dans la pharmacopée française. La théophylline (Euphylline $\AA$, Théostat $\AA$ ) est un inhibiteur non spécifique des différentes phosphodiestérases. C'est un bronchodilatateur moins puissant que les ß2-agonistes. Elle a également d'autres actions comme l'augmentation de la clairance mucociliaire, l'amélioration de la contraction musculaire striée (diaphragme) et la stimulation de la respiration. Elle a également des effets anti-inflammatoires. La voie d'administration de la théophylline dépend de l'indication. La forme à libération prolongée (per os) constitue actuellement en pratique la seule utilisation de ce produit. Elle est de moins en moins utilisée en raison de sa toxicité et de ses difficultés d'utilisation (taux sanguin efficace proche du taux toxique et interactions médicamenteuses).

- Les anticholinergiques ou les atropiniques de synthèse sont des antagonistes des récepteurs muscariniques. Ils sont bronchodilatateurs, mais moins puissants que les B2-agonistes. Les principaux produits sont le bromure d'ipratropium (Atrovent ${ }^{\circledR}$ ) et le bromure d'oxitropium (Tergisat ${ }^{\circledR}$ ). Ils ont une action bronchodilatatrice qui n'est pas immédiate (délai de $20 \mathrm{mn}$ ) d'où leur indication pour le traitement de fond de l'asthme sévère, instable, corticodépendant.

\section{PRISE EN CHARGE EN ODONTO- LOGIE}

\section{Le bilan médical}

Dans notre spécialité, en général, la chirurgie est réglée ce qui laisse suffisamment le temps de réaliser une évaluation préopératoire de la pathologie asthmatique du patient. Le patient 
ayant des antécédents d'asthme et un traitement en cours, doit apporter des informations précises sur l'état d'équilibre ou non de son asthme. L'appréciation de la gravité de l'asthme est de la compétence du spécialiste médical (pneumologue ou allergologue). Cette évaluation est indispensable pour guider la prise en charge préopératoire du patient. Elle repose sur le recueil d'un certain nombre de paramètres cliniques, biologiques et fonctionnels, obtenus sur une période suffisamment longue. Parmi ces paramètres, il faut surtout s'intéresser aux suivants : le nombre de crises d'asthme par jour, par semaine, par mois, la survenue diurne ou nocturne, la durée et la qualité des intervalles libres, l'éventualité de crises violentes imposant un recours médical immédiat, y compris l'hospitalisation, l'évolution des paramètres fonctionnels respiratoires (VEMS, DEP), le traitement prescrit et son observance. Ces divers paramètres permettront de classer le patient dans une des catégories définie par la classification internationale et de proposer en conséquence un traitement adapté (voir tableau 1). Dans la grande majorité des cas, les patients sont porteurs d'un asthme stable, bien contrôlé par le traitement médical et ne posant pas de problème.

La découverte d'un asthme instable, particulièrement à risque en période péri-opératoire, constitue une réelle contre-indication à toute chirurgie buccale. L'asthme instable est un asthme actif (variabilité du DEP, crises nocturnes motivant l'appel du médecin) imposant une adaptation du traitement préopératoire [13]. L'existence confirmée d'un asthme de stade 4 doit attirer la vigilance de l'odontologiste. La fréquence des crises étant importante chez ces patients il convient d'intervenir exclusivement en milieu hospitalier pour faire face à une crise sévère éventuelle.

De son coté, le chirurgien buccal doit mener un interrogatoire précis à la recherche d'une notion de dyspnée sifflante plus ou moins paroxystique. Nombreux encore sont les patients refusant de traiter leur asthme. Devant toute symptomatologie évocatrice, il convient d'adresser le malade au pneumologue pour confirmation du diagnostic et prise en charge thérapeutique.

\section{Préparation psychique à l'interven- tion chirurgicale en odontologie}

Le stress représente un des facteurs susceptibles de déclencher une crise d'asthme $[14,15,16]$. Pour le patient, la perspective de devoir subir une intervention de chirurgie buccale est souvent angoissante. II est préconisé d'intervenir de préférence le matin, avec des gestes peu traumatiques, dans un ambiance calme, avec des séances de courte durée. La préparation psychique du patient à l'acte chirurgical passe tout d'abord par une information sur la nature de l'acte, son déroulement et les suites opératoires, donnée dans un langage adapté. La consultation initiale doit permettre d'établir une relation de confiance qui détermine les conditions d'abord psychique pour l'intervention ultérieure. Dans un certain nombre de cas, en plus de la relation praticien-patient, le recours à une prescription médicamenteuse sédative est impérative.

\section{Conduite de l'anesthésie (locale ver- sus générale)}

II convient de préférer, chaque fois que cela est possible, l'anesthésie locale ou loco-régionale à l'anesthésie générale avec intubation, car le risque de bronchospasme est plus faible. L'anesthésie générale peut induire des complications broncho-pulmonaires se traduisant par un encombrement bronchique, une atélectasie ou une surinfection. La morbidité de l'anesthésie générale reste cependant faible du fait de l'utilisation d'agents anesthésiques, comme les halogénés, qui sont de puissants bronchodilatateurs [13].

L'anesthésie loco-régionale, bien que favorable en terme de risque opératoire, n'est pas un acte anodin pour le patient atopique. L'atopie, définie comme l'aptitude d'un sujet à se sensibiliser vis-à-vis des allergènes de l'environnement, représente un des principaux facteurs de risque de l'asthme. Chez l'enfant, la majorité des médecine

buccale

chirurgie

buccale

VOL. $9, \mathrm{~N}^{\circ} 1$ 2003

page 43 
médecine

buccale

chirurgie

buccale

VOL. $9, \mathrm{~N}^{\circ} 1$ 2003

page 44 asthmes ont une composante atopique, et le pourcentage diminue avec l'âge. Les anesthésiques locaux peuvent induire un bronchospasme, le plus souvent dû aux excipients présents dans la solution, et notamment les sulfites et les parabens. Les accidents anaphylactiques aux anesthésiques locaux sont exceptionnels et la confusion entre accident allergique et accident toxique est fréquemment constatée. Devant toute suspicion d'allergie, une consultation en allergologie est demandée. Des examens complémentaires (prick-tests ou intra-dermo-réactions) sont effectués pour investiguer cette allergie [17].

Les sulfites sont retrouvés dans toutes les solutions anesthésiques avec vasoconstricteur utilisées en odontologie puisque, en dehors de leur action anti-microbienne, ils jouent un rôle de conservateur, d'anti-oxydant pour les agents vasoconstricteurs. L'évolution n'a fait que multiplier leur utilisation industrielle, en particulier dans l'agro-alimentaire (cidre, bière, poissons séchés, fruits secs, salades sous cellophane), la cosmétique et la pharmaceutique. L'hypersensibilité et l'hyperréactivité aux métabisulfites (MBS) chez les asthmatiques sont des phénomènes reconnus depuis quelques années $[18,19]$. Les patients réagissent aussi bien aux MBS ingérés, inhalés et surtout injectés [20,21]. La crise d'asthme peut être initiée par des doses minimes de sulfites inférieures à $1 \mathrm{mg}$, dose rapidement atteinte lors d'infiltrations d'anesthésique multiples au cours d'une même séance. Pour certains auteurs, l'utilisation de vasoconstricteurs est strictement contre-indiquée alors que pour d'autres la contre-indication n'est pas aussi absolue. II est admis que 4 à $5 \%$ des asthmatiques présentent une intolérance aux sulfites et que, dans cette population, $40 \%$ d'entre eux ont un asthme cortico-dépendant [22]. La contre-indication des anesthésiques avec vasoconstricteurs ne concernerait que les asthmatiques corticodépendants. II faut rappeler également qu'une manifestation d'allergie aux sulfites peut apparaître d'emblée. L'utilisation de tests de provocation n'apporte pas de sécurité absolue puisqu'ils ont une faible sensibilité.
Les méthylparabens sont des excipients à visée bactériostatique et antifongique. Ces molécules ont un fort pouvoir allergisant et sont potentiellement inductrices de crises d'asthme. Les solutions anesthésiques contenant des méthylparabens sont contre-indiquées chez les patients asthmatiques.

Le ministère de la Santé et la Direction générale de la Pharmacie font obligation aux fabricants depuis septembre 1988, de mentionner clairement la teneur en sulfites $\mathrm{SO} 2$ contenus dans leurs produits. La Food and Drog Administration précise que les réactions d'intolérance peuvent être observées à partir de 0,6 à 0,9 mg de sulfites et, par conséquent, qu'il convient d'en tenir compte lors d'infiltrations multiples d'anesthésique chez un patient à risque comme le patient asthmatique. Pour l'asthmatique non corticodépendant, l'adrénaline représente le produit de choix car elle agit à la fois sur les récepteurs a et $B$. Cette action sur les récepteurs $B$ entraîne une bronchodilatation, favorable pour le patient asthmatique. Aux doses habituelles utilisées, cet effet $B$ reste cependant rarement décelable, sauf lors d'une injection intra-vasculaire accidentelle. A titre indicatif, la solution anesthésique la plus intéressante actuellement sur le marché est la Primacaïne $\AA$, sel d'articaïne adrénaliné au $1 / 200000(0,31 \mathrm{mg} / \mathrm{ml}$ de SO2 soit $0,527 \mathrm{mg}$ par carpule de $1,7 \mathrm{ml}$ ).

La contre-indication d'un vasoconstricteur impose de choisir une molécule avec des propriétés vasoconstrictrices intrinsèques : la molécule la plus vasoconstrictrice est représentée par la mépivacaïne (Scandicaïne $₫ 3 \%$ sans vasoconstricteur).

Dans tous les cas, l'injection de l'anesthésique local doit être réalisée très lentement, en s'assurant de l'absence d'injection intravasculaire (seringue aspirante), dans des tissus normalement vascularisés et non inflammatoires.

\section{Prescriptions médicamenteuses}

La prémédication sédative semble judicieuse chez le patient asthmatique pour lequel le contexte émotionnel et l'hyperventilation liée à l'angoisse peuvent initier la crise d'asthme 
aiguë. La problématique est que tous les sédatifs, même les anxiolytiques, sont des dépresseurs respiratoires. II convient de juger d'une part du degré d'anxiété pré-opératoire et de la difficulté de l'acte à réaliser pour choisir la prémédication la mieux adaptée. L'hydroxyzine (Atarax ${ }^{\circledR}$ ), à dose modérée, semble particulièrement convenir car cette molécule possède en plus de ses propriétés anxiolytiques, des effets antihistaminiques et anticholinergiques [15].

La prescription d'antibiotiques peut présenter un risque chez l'asthmatique ayant un terrain atopique. II est de règle de ne prescrire des $B$ lactamines, et notamment les pénicillines, qu'après avoir vérifié à l'interrogatoire l'absence d'allergie à cette famille d'antibiotique. Les macrolides sont utilisables, à l'exception de l'érythromycine si le patient bénéficie d'un traitement de fond à base de théophylline. En effet, des accidents de surdosage responsables de convulsions, de troubles cardiaques et de troubles de la conscience peuvent apparaître car l'érythromycine retarde l'élimination de la théophylline. Dans l'hypothèse d'un traitement de fond à base de théophylline et d'antécédents allergiques aux b-lactamines, la prescription anti-infectieuse peut faire appel à un macrolide pour lequel il n'y a pas d'interaction médicamenteuse - la spiramycine (Rovamycine $($ )), la synergistine (Pyostacine $\AA$ ) - ou à la clindamycine (Dalacine $\left.{ }^{\circledR}\right)$.

La prescription d'antalgiques doit privilégier les produits à base de paracétamol. Les antalgiques de niveau 2 (codéine, dextropropoxyphène) sont contre-indiqués car ces molécules favorisent la dépression respiratoire lors de la survenue d'une dyspnée aiguë.

La prescription d'anti-inflammatoires non stéroïdiens (AINS) doit être évitée de manière systématique si l'asthme a une composante allergique. Les AINS sont souvent prescrits après avulsion dentaire dans un but antalgique. Or ces substances peuvent être responsables d'effets secondaires liés à l'action anti-inflammatoire de la molécule : elles entraînent une augmentation importante du taux des leuco- triènes $\left(\mathrm{B}_{4}, \mathrm{C}_{4}, \mathrm{D}_{4}\right.$ et $\left.\mathrm{E}_{4}\right)$ et particulièrement des lencotriènes $C_{4}$ et $D_{4}$, qui sont de puissants bronchoconstricteurs. La prescription d'AINS peut déclencher une crise subintrante ou un état de mal asthmatique [11]. Pour tout acte chirurgical on doit privilégier une corticothérapie courte en pré-opératoire avec une molécule sans sulfites comme la prednisolone (Solupred $(\AA)$ ) et éliminer la bêtaméthasone (Célestène $\AA$ ) renfermant des sulfites. Pour une corticothérapie d'urgence, on préfère pour les mêmes raisons la méthylprednisolone (Solumédrol $®$ ) à la dexaméthasone (Soludécadron $\AA$ ) contenant des sulfites (0,62 mg/ampoule de $1 \mathrm{ml})$. Lorsque le patient est traité au long cours par une corticothérapie orale ou par aérosol, le traitement doit être poursuivi. La programmation d'une intervention chirurgicale n'impose pas systématiquement une modification de la posologie. Enfin, dans le contexte d'un asthme instable, le traitement corticoïde par voie orale ou intraveineuse peut interférer avec le processus cicatriciel et augmenter le risque infectieux [13].

\section{Exposition aux pneumallergènes}

L'odontologiste doit veiller à réduire les poussières de fraisage (émail et dentine) qui peuvent constituer les facteurs déclenchants d'une crise d'asthme par leur action irritative mécanique. En odontologie chirurgicale, l'utilisation de la turbine est proscrite, car elle génère un spray qui de plus est septique. L'utilisation de moteurs chirurgicaux tournant à 40000 tours/ minute associée à une puissante aspiration chirurgicale constitue un gage de sécurité.

Le latex constitue un pneumallergène violent pour le sujet asthmatique. L'hypersensibilité au latex est devenu un sujet d'actualité. Les particules d'amidon présentes sur la surface des gants peuvent agir comme irritant et provoquer un bronchospasme, une crise d'asthme ou une détresse respiratoire [23-25]. Les patients atopiques sont beaucoup plus exposés au risque de développer une allergie au latex [24]. Les manifestations cliniques respiratoires de cette hypersensibilité peuvent être induites par simple contact cutanéo-muqueux ou par inhala- médecine

buccale

chirurgie

buccale

VOL. $9, \mathrm{~N}^{\circ} 1$ 2003

page 45 
médecine buccale chirurgie buccale

VOL. $9, \mathrm{~N}^{\circ} 1$ 2003

page 46 tion de particules de latex présentes dans l'air ambiant. Lorsqu'il existe des antécédents d'allergie au latex, le praticien préconisera une alternative par des produits de substitution comme les gants stériles en nitrile et s'assurera de la bonne aération de la salle d'intervention $[26,27]$.

\section{Conduite à tenir face à une crise d'asthme en cours de soins}

La survenue d'une crise d'asthme au cours des soins reste peu fréquente si on respecte les mesures préventives. A ce titre, il est demandé au patient de venir avec son spray de B2-agoniste pour l'intervention. Certains praticiens ordonnent à titre préventif deux inhalations de bronchodilatateurs avant les soins ainsi qu'une corticothérapie flash à faible dose 24 heures avant l'acte (20 mg de prednisolone) pour prévenir la crise ; si elle survient néanmoins, les secrétions bronchiques sont alors moins importantes.

La survenue d'une crise d'asthme en per-opératoire impose l'arrêt immédiat des soins et la mise en route d'un traitement d'urgence, en raison de l'évolution potentielle vers une détresse respiratoire aiguë. Le praticien s'assure de la vacuité de la cavité buccale et installe le patient en position demi-assise, pour faciliter sa respiration. Le premier geste thérapeutique consiste à administrer deux bouffées de B2-agoniste (Ventoline,...) grâce à une chambre d'inhalation qui présente l'avantage d'augmenter le dépôt pulmonaire, de réduire le dépôt oropharyngé et de diminuer la taille des particules favorisant alors la pénétration pulmonaire. Une évolution favorable doit être observée en quinze minutes. II est possible de répéter l'opération plusieurs fois si le résultat initial n'est pas suffisant, à raison de deux inhalations toutes les dix-quinze minutes avec un maximum de 8 à 10 bouffées. Parallèlement aux inhalations, une oxygénothérapie au masque ou avec des lunettes est réalisée avec un débit de 6 à 9 I.min-1. L'évolution vers la crise d'asthme sévère ou l'asthme aigu grave, marquée par la persistance des symptômes malgré la prise de ß2-agonistes et l'oxygénothérapie, implique l'appel immédiat du SAMU. En attendant son arrivée, l'injection sous-cutanée de $0,5 \mathrm{mg}$ de terbutaline (Bricanyl ${ }^{\circledR}$ ) est préconisée.

L'asthme aigu grave correspond à une crise d'asthme de gravité inhabituelle pouvant mettre en jeu le pronostic vital. Les critères de gravité de cette crise sont bien codifiés $[4,28]$ et comportent les éléments consignés dans le tableau 3.

Tableau 3 : Critères de gravité d'une crise d'asthme d'après ROUE et coll [28].

Criteria of gravity of asthma attacks from ROUE and coll [28].

- Difficultés à parler - Orthopnée

- Agitation et sueurs

- Troubles de la conscience

- Cyanose

- Contraction des muscles sterno-cléido-mastoïdiens

- Bradycardie, collapsus

- Pauses respiratoires

- Fréquence respiratoire supérieure à 30 cycles.min-1

- Fréquence cardiaque supérieure à 130.min-1

- Normo- ou hypercapnie

Les autres mesures thérapeutiques dépendent des conseils du médecin régulateur renseigné sur l'évolution de la symptomatologie : administration de bronchodilatateur (Ventoline, ou Bricanyl $\circledast$ ) par voie sous-cutanée, intra-musculaire ou intra-veineuse en plus de l'oxygénothérapie au masque à 8 à $10 \quad 1 . \mathrm{min}^{-1}$, puis éventuellement utilisation de corticoïdes type Solumédrol, ou d'adrénaline. L'hospitalisation en soins intensifs spécialisés est de règle. La survenue d'un arrêt respiratoire ou circulatoire impose la mise en œuvre des mesures de réanimation : ventilation au masque et massages cardiaques externes jusqu'à l'arrivée du SAMU. 


\section{CONCLUSION}

La phase pré-opératoire constitue une étape capitale de la prise en charge du patient asthmatique en chirurgie buccale. Elle permet d'évaluer la sévérité de l'affection et de déterminer le type d'anesthésie adapté à la nature de l'intervention chirurgicale, tout en tenant compte des caractéristiques propres à chaque patient. La mise en évidence d'un asthme instable doit conduire à différer l'acte et à adop-

\section{RÉFÉRENCES}

1 - TAYrard A. Qu'en est-il en 1996 de la morbidité et de la mortalité induites par l'asthme? Rev Pneumol Clin 1996; 52: 65-9.

2 - Enquête ASUR (groupe d'étude sur AsthmeUrgences). Programme d'actions de prévention et de prise en charge de l'asthme. http://www.sante.gouv.fr.

3 - Salmeron S, Liard R, ElkHarrat D, Muir JF, NeUKIRCH F, ElLRODT A. Asthma severity and adequacy of management in accident and emergency departments in France: a prospective study. Lancet 2001; 358: 629-35.

4 - Jaffuel D, Demoly P, Moulaire V, Landreau L, Bousquet J, Michel FB, Jonquet O, Godard PH. Prise en charge thérapeutique de l'asthme aigu grave de l'adulte. Presse Med 1997; 26: 640-5.

5 - Pariente R. Asthme bronchique. Traitement de fond. Presse Med 1997; 26: 633-8.

6 - Michel FB, Chanez P, Godard PH, Bousquet J. Asthme bronchique. Physiopathologie. Conception classique et nouveaux concepts. Presse Med 1997; 26: 621-31.

7 - Sollecito TP, Tino G. Asthma. Oral Surg Oral Med Oral Pathol Oral Radiol Endod 2001; 92: 485-90.

8 - Georgitis JW. The 1997 Asthma Management Guidelines and therapeutic issues relating to the treatment of asthma. National Heart, Lung, and Blood Institute. Chest 1999; 115: 210-7.

9 - Godard P, Chanez P, Bousquet J. Etude analytique des différents traitements (pp230-255) In: Asthmologie. éd : Masson, Paris, 1996.

10 - Gut-Cobert C, Chenu E, Lemière C, Leroyer C. Actualités de la prise en charge de la maladie asthmatique. Presse Med 2000; 29: 761-5.

11 - LOCKHART A, DINH-XUAN AT. Physiopathologie de l'asthme : quelle place pour les leucotriènes ? Rev Pneumol Clin 1997; 53: 119-27. ter avec le pneumologue une stratégie commune. Hormis le cas d'asthme instable, la prise en charge du patient asthmatique ne doit pas poser de problème majeur si, en plus de cette évaluation pré-opératoire, le chirurgien buccal respecte les mesures préventives per- et postopératoire, et s'il connaît les symptômes et la conduite à tenir devant un bronchospasme qui peut toujours survenir malgré le respect des mesures préventives.

12 - Devillier PH, Bessard G, Advenier CH. Les antagonistes des leucotriènes: une nouvelle approche du traitement de l'asthme. Rev Mal Resp 1997; 14: 159-70.

13 - Dureuil B, Maitrepierre C, Matuszczak Y. Prise en charge péri-opératoire des sujets à risque : les asthmatiques. Presse Med 1998; 27: 437-42.

14 - Adel N, Dutau H, Gouitaa M, Charpin D. Facteurs de risque de l'asthme grave. Rev Mal Resp 1998; 15: 683-97.

15 - DeREK M, Steinbacher DM, GLick M. The dental patient with asthma. An update and oral health considerations. J Am Dent Assoc 2001; 132: 1229-39.

16 - Mathew T, Casamassimo PS, Wilson S MA, Preisch J, Allen E, HAYES JR. Effect of dental treatment on the lung funtion of children with asthma. J Am Dent Assoc 1998; 129: 1120-8.

17 - LeYNADIER F. Du bon usage des anesthésiques locaux. Actual Odontostomatol 1992; 179: 481-5.

18 - SENG GF, GAY BJ. Dangers of sulfites in dental local anesthetic solutions: warning and recommendations. J Am Dent Assoc 1986; 113: 769.

19 - Gumowski PI, Huguenin A, Bernard JP, Angelone T, FIORE-DONNO, GIRARD JP. Réactions secondaires aux anesthésiques locaux : rôle possible des métabisulfites. Med Hyg 1988; 46: 3168-75.

20 - Miltgen J, Marotel C, Natali F, Vaylet F, L'her P. Aspects cliniques et diagnostic de l'intolérance aux sulfites. Revue Pneumol Clin 1996; 52 : 363371.

21 - Maria Y, Vaillant P, Delorne N, Moneret-Vautrin DA. Les accidents graves liés aux métabisulfites. Rev Med Inter 1989;10: 36-40.

22 - Guérin T, LAURENT P, WierzBA CB. Le patient asthmatique en milieu odontologique. Actual Odontostomatol 2000; 210: 195-206. \begin{tabular}{l}
$\begin{array}{l}\text { médecine } \\
\text { buccale } \\
\text { chirurgie } \\
\text { buccale }\end{array}$ \\
\hline VOL. $9, N^{\circ} 1$ \\
2003 \\
\hline page 47
\end{tabular} 
23- Aichane A, Bouayad Z, El Outmani A, Afif $H$, Trombati N, Bahlaoul A. Allergie au latex en milieu hospitalier. Rev Mal Respir 1997;14: 451-5.

24 - Garabant DH, Roth DH, PARSAD R, Ying GS, WeISS J. Latex sensitization in health care workers and in the US general population. Am J Epidemiol 2001; 153: 515-22.

25 - LeYnADIER F. Allergie au latex : les gants poudrés doivent être interdits ! Rev Prat 2002; 16: 345-8.
26 - Ely G. Irritations, allergies, infections, asthme : les gants d'examen en question, la poudre facteur de risque. Inf Dent 2002; 12: 751-62.

27 - NATTRASS C, IRELAND AJ, LOVELL CR. Latex allergy in an orthognathic patient and implications for clinical management. Br J Oral Maxillofac Surg 1999; 37:11-3.

28 - Roue C, Fournier M, Sleiman CH, Mal H, Pariente R. Traitement de l'asthme aigu grave. Rev Pneumol Clin 1996; 52 :129-34. médecine

buccale

chirurgie

buccale

VOL. $9, \mathrm{~N}^{\circ} 1$

2003

page 48 